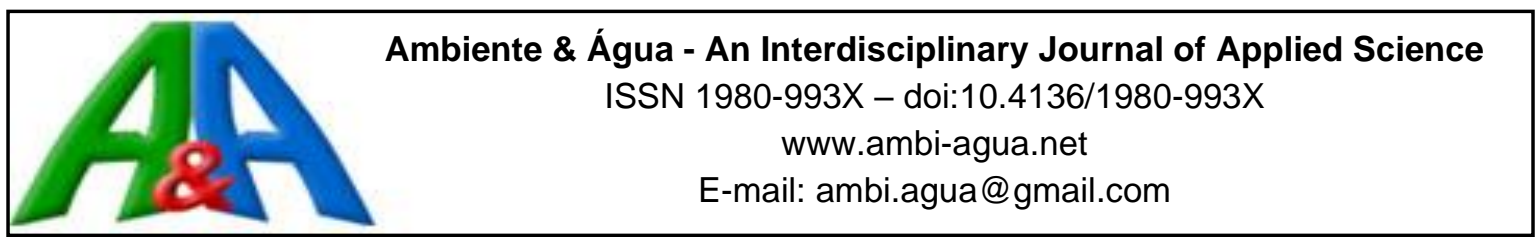

\title{
Distribution of major and trace elements in bottom sediments of the Taquari River Basin, Caldas municipality (Brazil)
}

\author{
ARTICLES doi:10.4136/ambi-agua.2397 \\ Received: 26 Mar. 2019; Accepted: 25 Jun. 2019
Pedro Henrique Dutra ${ }^{*}$; ; Vanusa Maria Delage Feliciano1 ${ }^{1}$; Carlos Alberto De Carvalho Filho 1 ic
${ }^{1}$ Departamento de Serviço do Meio Ambiente, Centro de Desenvolvimento da Tecnologia Nuclear (CDTN), Avenida Presidente Antônio Carlos, nº 6.627, CEP 31270-901, Belo Horizonte, MG, Brazil.
E-mail: feliciaovanusa@gmail.com, calbertocf@gmail.com
*Corresponding author. E-mail: pedrohenrique.dutra@gmail.com

\begin{abstract}
The Taquari River Basin, located in Poços de Caldas Alkaline Complex, in the southern portion of Minas Gerais state, Brazil, is situated in an old volcanic caldera. Due to its chemical and radiological characteristics, it is an area of economic and mineral interest, and is also home to diverse flora and fauna systems. In its surroundings, there are agricultural areas, industries (active and inactive) and urban and rural centers. This work investigated the total and potentially bioavailable concentrations of major and trace elements for the evaluation of geogenic and anthropogenic contamination potentials in the water bodies. The results show that there is an anthropogenic contribution (fertilizers and mining tailings) in some sectors of the Taquari River Basin, generating possible concerns regarding the quantity of elements that may be transferred to the water bodies. Furthermore, there is the striking geogenic contribution from naturally enriched areas, presenting distinct situations that generate an increase in the concentration of chemical elements in the water bodies.
\end{abstract}

Keywords: fertilizers, sediments quality, uranium mine wastes.

\section{Distribuição de elementos maiores e traços em sedimentos de fundo da Bacia do Rio Taquari, município de Caldas (Brasil)}

\section{RESUMO}

A Bacia do Rio Taquari, localizada no Complexo Alcalino de Poços de Caldas, na porção sul de Minas Gerais, está situada em uma antiga caldeira vulcânica. Devido às suas características químicas e radiológicas, é uma área de interesse econômico e mineral, além de abrigar diversos sistemas de flora e fauna. Nos seus arredores existem áreas agrícolas, indústrias (ativas e inativas) e centros urbanos e rurais. O objetivo deste trabalho foi investigar as concentrações totais e potencialmente biodisponíveis de elementos principais e traços para a avaliação de potenciais de contaminação geogênica e antropogênica em corpos d'água. Os resultados mostram que há uma contribuição antrópica (fertilizantes e rejeitos de mineração) em alguns setores da bacia do rio Taquari, gerando possíveis preocupações quanto à quantidade de elementos que podem ser transferidos para os corpos hídricos. Além disso, há contribuição geogênica de áreas naturalmente enriquecidas, apresentando situações distintas que geram um aumento na concentração de elementos químicos nos corpos d'água.

Palavras-chave: fertilizantes, qualidade dos sedimentos, rejeitos de mina de urânio. 


\section{INTRODUCTION}

Sites that present areas contaminated by agrochemicals (EMBRAPA, 2004) or by industrial effluents can have, for the most part, soils with high metal contents, affecting sustainability, biodiversity and productivity, and offering a high degree of danger to the population (Sun et al., 2001). Mining activities raise various environmental impacts depending on their size, exploitation and processing methods employed. These potential impacts directly or indirectly affect the local environment and communities and, in some cases, also the surrounding area (Marnika et al., 2015). In the mineral industry generally, a large amount of tailings is generated, mainly in the steps of mining and the chemical processing of the ore, generally deposited in piles and tailing dams, respectively. Effluents from both tailings can carry toxic substances and seriously impact the environment. The presence of sulfides in tailings can lead to acid mine drainage, considered a serious problem in mining projects, due to the degradation of the quality of groundwater and surface waters, as well as soils and sediments (USEPA, 1994)

The systematic and often inadequate use of compounds for soil correction can result in increased accumulation of metals and toxic substances in soils, which may migrate to groundwater, surface water and river beds, often in concentrations that can cause severe impacts to the environment. The river bottom sediments are fundamental components of the river environment, which provide nutrients for living organisms and act as receptors of anthropogenic contaminants (Reis et al., 2010). Studies on the evaluation of environmental impacts due to the use of agricultural correctives have been developed throughout the world and in Brazil (Savci, 2012; Yousaf et al., 2017). One aspect to consider in agricultural/industrial activities is rock phosphates. These constitute the main raw material for manufacture of phosphate fertilizers. During the production process, most metals remain in fertilizers (Freitas et al., 2009; Valle, 2012).

It is known that these phosphates naturally contain several metals, which may be of concern with regards to environmental contamination (Agbenin, 2002). It is important to mention some studies that indicate uranium (U) and thorium (Th) contents and their decay products in phosphate fertilizers, which, depending on the origin of the phosphate rock, may be significant (Saueia and Mazzilli, 2006; Jacomino et al., 2009). The main objective of the present study was to evaluate if anthropogenic and/or geogenic agents are contributing to the increase of the concentration of certain metals in the fluvial sediments of the study area.

The study area extends for $102 \mathrm{~km}^{2}$ and is located in the municipality of Caldas, in the central-southeast region of the Poços de Caldas Alkaline Complex (PCAC) (Figure 1). The area is located in the Taquari River Basin, consisting of the Consulta and Soberbo Streams and the Taquari River itself. The main types of soil use and occupation of the Taquari River Basin are agriculture and the Caldas Uranium Mine (CUM), which is currently in the decommissioning process (Figure 1). Although no longer in production, previous works (Fernandes et al., 1995; Carvalho Filho et al., 2016; 2017) in the area surrounding this former mine indicate possibilities for the generation of liabilities and contamination of the external environment, mainly as a consequence of acid mine drainage (AMD).

The agricultural sector is represented in the study area by vegetables, dairy and cattle farming, ornamental plants (mainly roses) and silviculture (with greatest expansion in the last 20 years). This plantation growth has generated a change in the local soil chemistry, resulting from agrochemicals, fertilizers and soil correctives. Continuous application together with the high turnover can generate undue accumulations of non-essential elements in the soil and later in water bodies.

The local geology comprises a set of plutonic, volcanic and subvolcanic rocks belonging to the PCAC (Figure 1). Nepheline syenites, phonolites, volcanic breccias and pyroclastic rocks 
predominate in the area (Schorscher and Shea, 1992; Valeton et al., 1997). The CUM uranium deposit is defined as a mineralization of U-Th-Zr-Mo-REE (rare earth elements), composed mainly of uranium black oxides (uraninite and pitchblende - $\mathrm{UO}_{2}$ ), which is occasionally accompanied by cofinite $\left(\mathrm{U}^{4+}, \mathrm{Th}\right)\left(\mathrm{SiO}_{4}\right)_{1-\mathrm{x}}(\mathrm{OH})_{4 \mathrm{x}}$. The mineralogy of the ore consists of: sulfide minerals, mainly pyrite $\mathrm{FeS}_{2}$ and rarely galena $\mathrm{PbS}$ and sphalerite $\mathrm{ZnS}$; zirconium minerals, generally zircon- $\mathrm{Zr} \mathrm{SiO} 4$; minerals containing Mo, mainly jordisite $\mathrm{MoS}_{2}$ and ilsemanite $\mathrm{Mo}_{3} \mathrm{O}_{8} . \mathrm{nH}_{2} \mathrm{O}$; fluorite $\mathrm{CaF}_{2}$ and REE phases (Schorscher and Shea, 1992; Waber et al., 1992).

The Morro do Taquari U-Zr-anomaly is found in the study area (Figure 1) and was characterized by the presence of caldasite (CT), a complex ore of zircon $\left(\mathrm{ZrSiO}_{4}\right)$ and badeleite $\left(\mathrm{ZrO}_{2}\right)$ containing uranium inclusions (Schorscher and Shea, 1992). The mining waste, consisting of barren rocks and low-grade ore, were added to dump deposits or bota-fora (BF), among which bota-fora 4 (BF4; Figure 1) is the most important from an environmental point of view. The wastes from the chemical treatment and acid neutralization were deposited in the tailings dam (BR; Figure 1), and after the addition of $\mathrm{BaCl}_{2}$ to force radio precipitation, the effluent from the BR pour into the Soberbo Stream. The oxidation of pyrite in the wastes of BF4 and BR produces AMD, which when percolating through the waste can dissolve uranium and other toxic metals present.

Studies performed in the region of the uranium mine (Fernandes et al., 1995; 1998; Carvalho Filho et al., 2016; 2017) show a migration of acid mine drainage to water bodies, which can result in surface water and groundwater contamination.
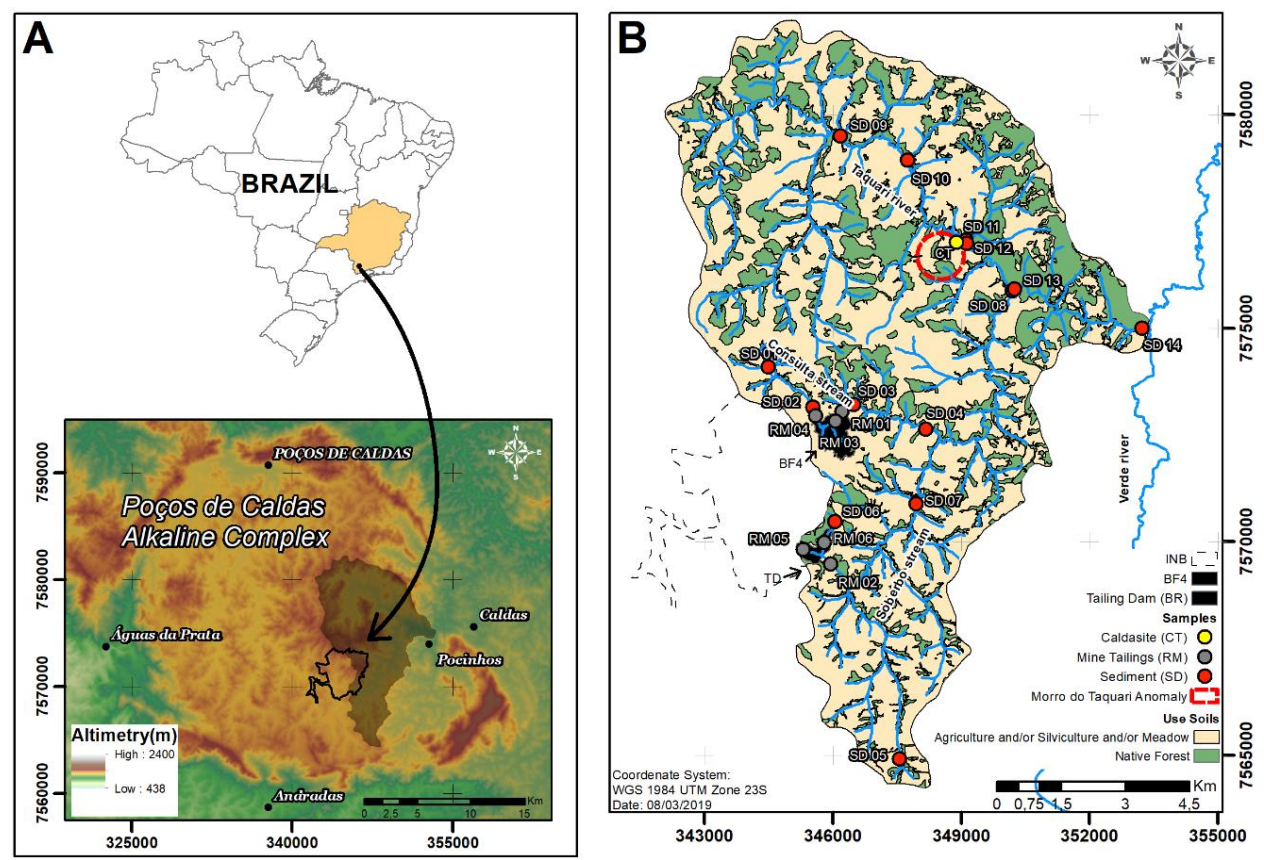

Figure 1. A) Location map of the study area. Source: Modified from Carvalho Filho et al. (2016); B) the sampling stations and soil use and occupation map.

Source: research data.

\section{MATERIALS AND METHODS}

The methodology of the study was structured considering the following: a) the potential anthropogenic sources of contamination are represented by fertilizers and CUM tailings, classified as diffuse and point sources, respectively; b) The caldasite ore was considered as a geogenic source; c) Fluvial sediments from the Taquari River Basin represent the matrix under investigation. 
A total of 14 fluvial sediment samples (SD) were collected (Figure 1): six upstream of the CUM discharges - SD01, SD05, SD09, SD10 SD11 and SD12. The last two are a few meters downstream of the Morro do Taquari U-Zr-anomaly; the others are downstream of CUM. All fluvial samples are in places where there may be fertilizer application. A total of six mining tailings samples were collected (RM, Figure 1): 3 in BF4 (RM01, RM03 and RM04) and 3 in BR (RM02, RM05 and RM06).

The procedure for collecting the sediment and mining tailing samples was performed by using a shovel or a device for removing material from the river bottom, called a "rock-islander". Selection of fertilizers was done in conjunction with the "Company of Technical Assistance and Rural Extension of the State of Minas Gerais" (EMATER), where seven samples (FT01 to FT07) were collected from farms near the sediment sampling points. For the geogenic species, a composite sample of caldasite ores (CT) was prepared from 10 single random samples collected in outcrops at the Morro do Taquari anomaly (Figure 1).

The sediment and tailing samples were dried at a temperature of approximately $80^{\circ} \mathrm{C}$, then disaggregated and sieved, obtaining an approximate fraction of $<63 \mu \mathrm{m}$ for identification and quantification of the elements present. Caldasite was crushed and pulverized $(63 \mu \mathrm{m})$. The fertilizer samples were only pulverized $(63 \mu \mathrm{m})$.

To quantify the elements $\mathrm{Al}, \mathrm{Ca}, \mathrm{Fe}, \mathrm{K}, \mathrm{Mg}, \mathrm{Mn}, \mathrm{Na}, \mathrm{P}, \mathrm{Pb}, \mathrm{Rb}, \mathrm{Si}, \mathrm{Sr}$, Ti, $\mathrm{Zn}$ and $\mathrm{Zr}$, the powdered samples underwent a press and compaction process with boric acid. Measurements of this material were performed using an X-ray fluorescence spectrometer, brand Rigaku, model ZSX Primus II, at the Mineral Technology Service of the Nuclear Technology Development Center (CDTN).

Uranium and thorium were analyzed at Analytical Chemistry and Radiochemistry Service of the CDTN, by the neutron activation technique, thorium by the k0-AAN method (Menezes and Jacimovic, 2006) and uranium by the delayed fission neutrons method (Jacomino et al., 2009). The samples were irradiated using the CDTN's nuclear research reactor TRIGA MARK I IPR-R1 and measured by a Canberra gamma spectrometry system, coaxial model 5019 HPGe detector with $50 \%$ nominal efficiency.

The Enrichment Factor (EF) is an index that evaluates the influence of metal sources of anthropogenic and geogenic origin in sediments (Selvaraj et al., 2004), and is defined as Equation 1:

$$
E F=\frac{\left[\frac{C_{M} \text { sample }}{C_{N E} \text { sample }}\right]}{\left[\frac{C_{M} \text { reference }}{C_{N E} \text { reference }}\right]}
$$

$\mathrm{C}_{\mathrm{M}}$ sample is the total concentration of metal $\mathrm{M}$ in the sample; $\mathrm{C}_{\mathrm{NE}}$ sample is the total concentration of the normalizing element in the sample; $\mathrm{C}_{M}$ reference is the total concentration of element $\mathrm{M}$ in the reference standard; $\mathrm{C}_{\mathrm{NE}}$ reference is the total concentration of the normalizing element in the reference standard. For calculating the enrichment factor, the elements $\mathrm{Fe}, \mathrm{Al}$ and $\mathrm{Sc}$ are usually adopted as normalizing elements (NE) (Schropp et al., 1990; Din, 1992). In the present study, iron was selected as NE, and as reference standard were used the mean values of elemental concentrations found by Valeton et al. (1997) for the PCAC rocks.

EF values of approximately 1.0 indicate that the metal in the analyzed sediment is predominantly of geogenic origin, contrary to values greater than 1 which indicates that the metal is of anthropogenic origin. Chen et al. (2007) proposed a degree of enrichment based on $\mathrm{EF}$ values: $\mathrm{EF}<1$, no enrichment; $1<\mathrm{EF} \leq 3$, little enrichment; $3<\mathrm{EF} \leq 5$, moderate enrichment; $5<\mathrm{EF} \leq 10$, moderately severe enrichment; $10<\mathrm{EF} \leq 25$, severe enrichment, $25<\mathrm{EF} \leq 50$ very severe enrichment, $>50$, extremely severe enrichment. 


\section{RESULTS AND DISCUSSION}

Table 1 shows the elemental concentrations found in sediment (research data) and local rock (Valeton et al., 1997).

Table 1. Fluvial sediment concentrations (SD) and reference standard local rock (LR).

\begin{tabular}{|c|c|c|c|c|c|c|c|c|c|c|}
\hline \multirow[b]{2}{*}{ Samples } & \multicolumn{10}{|c|}{ ELEMENTS (\%) } \\
\hline & $\mathrm{Al}$ & $\mathrm{Ca}$ & $\mathrm{Fe}$ & K & $\mathrm{Mg}$ & $\mathrm{Mn}$ & $\mathrm{Na}$ & $\mathrm{P}$ & $\mathrm{Si}$ & $\mathrm{Ti}$ \\
\hline SD01 & $20.01 \pm 0.10$ & $0.06 \pm 3 \times 10^{-4}$ & $3.85 \pm 0.01$ & $3.03 \pm 0.01$ & $0.07 \pm 3 \times 10^{-4}$ & $0.04 \pm 2 \times 10^{-5}$ & $0.01 \pm 5 \times 10^{-6}$ & $0.31 \pm 1 \times 10^{-4}$ & $22.81 \pm 0.11$ & $1.08 \pm 0.005$ \\
\hline SD02 & $19.85 \pm 0.09$ & $0.04 \pm 2 \times 10^{-4}$ & $4.20 \pm 0.02$ & $5.56 \pm 0.02$ & $0.08 \pm 4 \times 10^{-4}$ & $0.27 \pm 1 \times 10^{-4}$ & $0.04 \pm 2 \times 10^{-5}$ & $0.18 \pm 9 \times 10^{-5}$ & $21.50 \pm 0.10$ & $0.78 \pm 0.003$ \\
\hline SD03 & $18.68 \pm 0.09$ & $0.03 \pm 2 \times 10^{-4}$ & $4.34 \pm 0.02$ & $6.72 \pm 0.03$ & $0.07 \pm 3 \times 10^{-4}$ & $0.39 \pm 2 \times 10^{-4}$ & $0.03 \pm 1 \times 10^{-5}$ & $0.11 \pm 5 \times 10^{-5}$ & $21.46 \pm 0.10$ & $0.60 \pm 0.003$ \\
\hline SD04 & $18.56 \pm 0.09$ & $0.24 \pm 1 \times 10^{-4}$ & $1.92 \pm 0.01$ & $3.72 \pm 0.01$ & $0.08 \pm 4 \times 10^{-4}$ & $0.06 \pm 3 \times 10^{-5}$ & $0.50 \pm 2 \times 10^{-4}$ & $0.06 \pm 3 \times 10^{-5}$ & $20.04 \pm 0.10$ & $0.60 \pm 0.003$ \\
\hline SD05 & $21.59 \pm 0.10$ & $0.20 \pm 1 \times 10^{-4}$ & $7.55 \pm 0.03$ & $1.49 \pm 0.01$ & $0.21 \pm 1 \times 10^{-4}$ & $0.19 \pm 9 \times 10^{-5}$ & $0.02 \pm 2 \times 10^{-5}$ & $0.18 \pm 9 \times 10^{-5}$ & $19.02 \pm 0.09$ & $2.04 \pm 0.10$ \\
\hline SD06 & $21.70 \pm 0.10$ & $0.12 \pm 6 \times 10^{-5}$ & $4.76 \pm 0.02$ & $4.32 \pm 0.02$ & $0.10 \pm 5 \times 10^{-5}$ & $0.08 \pm 4 \times 10^{-5}$ & $0.05 \pm 2 \times 10^{-5}$ & $0.19 \pm 9 \times 10^{-5}$ & $20.05 \pm 0.10$ & $1.20 \pm 0.006$ \\
\hline SD07 & $18.03 \pm 0.09$ & $0.25 \pm 1 \times 10^{-4}$ & $4.85 \pm 0.02$ & $2.22 \pm 0.01$ & $0.17 \pm 1 \times 10^{-4}$ & $0.15 \pm 7 \times 10^{-5}$ & $1.33 \pm 6 \times 10^{-4}$ & $0.06 \pm 3 \times 10^{-5}$ & $16.54 \pm 0.08$ & $1.00 \pm 0.005$ \\
\hline SD08 & $17.65 \pm 0.08$ & $0.34 \pm 1 \times 10^{-4}$ & $4.57 \pm 0.02$ & $3.42 \pm 0.01$ & $0.63 \pm 3 \times 10^{-4}$ & $0.15 \pm 7 \times 10^{-5}$ & $0.16 \pm 1 \times 10^{-4}$ & $0.07 \pm 3 \times 10^{-5}$ & $17.85 \pm 0.08$ & $1.09 \pm 0.005$ \\
\hline SD09 & $19.16 \pm 0.09$ & $0.19 \pm 2 \times 10^{-4}$ & $4.83 \pm 0.02$ & $5.81 \pm 0.02$ & $0.10 \pm 5 \times 10^{-5}$ & $0.46 \pm 2 \times 10^{-4}$ & $0.11 \pm 5 \times 10^{-5}$ & $0.11 \pm 5 \times 10^{-5}$ & $21.27 \pm 0.10$ & $1.08 \pm 0.005$ \\
\hline SD10 & $23.39 \pm 0.11$ & $0.07 \pm 3 \times 10^{-5}$ & $4.90 \pm 0.02$ & $3.82 \pm 0.01$ & $0.11 \pm 5 \times 10^{-5}$ & $0.26 \pm 1 \times 10^{-4}$ & $0.03 \pm 1 \times 10^{-5}$ & $0.09 \pm 4 \times 10^{-5}$ & $18.74 \pm 0.09$ & $1.14 \pm 0.005$ \\
\hline SD11 & $19.58 \pm 0.09$ & $0.24 \pm 1 \times 10^{-4}$ & $4.34 \pm 0.02$ & $5.73 \pm 0.02$ & $0.11 \pm 5 \times 10^{-5}$ & $0.66 \pm 3 \times 10^{-4}$ & $0.10 \pm 5 \times 10^{-5}$ & $0.12 \pm 6 \times 10^{-5}$ & $20.89 \pm 0.10$ & $1.14 \pm 0.005$ \\
\hline SD12 & $21.12 \pm 0.10$ & $0.08 \pm 4 \times 10^{-5}$ & $5.81 \pm 0.02$ & $4.81 \pm 0.02$ & $0.11 \pm 5 \times 10^{-5}$ & $0.40 \pm 2 \times 10^{-4}$ & $0.04 \pm 2 \times 10^{-5}$ & $0.12 \pm 6 \times 10^{-5}$ & $19.73 \pm 0.09$ & $0.78 \pm 0.003$ \\
\hline SD13 & $17.65 \pm 0.08$ & $0.34 \pm 1 \times 10^{-4}$ & $4.56 \pm 0.02$ & $3.41 \pm 0.01$ & $0.14 \pm 5 \times 10^{-5}$ & $0.15 \pm 7 \times 10^{-5}$ & $0.23 \pm 1 \times 10^{-4}$ & $0.07 \pm 3 \times 10^{-5}$ & $17.85 \pm 0.08$ & $1.09 \pm 0.005$ \\
\hline SD14 & $17.56 \pm 0.08$ & $0.34 \pm 1 \times 10^{-4}$ & $4.52 \pm 0.02$ & $3.39 \pm 0.01$ & $0.27 \pm 1 \times 10^{-4}$ & $0.15 \pm 7 \times 10^{-5}$ & $0.24 \pm 1 \times 10^{-4}$ & $0.03 \pm 2 \times 10^{-5}$ & $17.73 \pm 0.08$ & $1.08 \pm 0.005$ \\
\hline \multirow[t]{2}{*}{$\mathrm{LR}$} & $17.36 \pm 0.08$ & $0.46 \pm 2 \times 10^{-4}$ & $3.42 \pm 0.01$ & $4.21 \pm 0.02$ & $0.09 \pm 4 \times 10^{-5}$ & $0.10 \pm 5 \times 10^{-5}$ & $2.22 \pm 1 \times 10^{-3}$ & $0.03 \pm 1 \times 10^{-5}$ & $18.24 \pm 0.09$ & $0.44 \pm 0.002$ \\
\hline & \multicolumn{10}{|c|}{ ELEMENTS $\left(\mathrm{mg}^{\prime} \mathrm{kg}^{-1}\right)$} \\
\hline Samples & $\mathrm{Ba}$ & $\mathrm{Cu}$ & $\mathrm{Ni}$ & $\mathrm{Pb}$ & $\mathrm{Rb}$ & $\mathrm{Sr}$ & Th & $\mathrm{U}$ & $\mathrm{Zn}$ & $\mathrm{Zr}$ \\
\hline SD01 & $<1$ & $<1$ & $<1$ & $176.4 \pm 0.88$ & $128.0 \pm 0.64$ & $803.3 \pm 4.01$ & $66.18 \pm 0.33$ & $10 \pm 0.05$ & $273.2 \pm 1.36$ & $1111 \pm 5.55$ \\
\hline SD02 & $447.8 \pm 2.24$ & $15.98 \pm 0.08$ & $<1$ & $557.0 \pm 2,78$ & $210.3 \pm 1.05$ & $1015 \pm 5.07$ & $118.7 \pm 0.59$ & $68 \pm 0.34$ & $128.5 \pm 0.64$ & $1851 \pm 9.25$ \\
\hline SD03 & $223.9 \pm 1.12$ & $<1$ & $<1$ & $185.7 \pm 0.92$ & $274.3 \pm 1.37$ & $651.1 \pm 3.25$ & $106.6 \pm 0.53$ & $210 \pm 1.05$ & $514.2 \pm 2.67$ & $2147 \pm 10.73$ \\
\hline SD04 & $1692 \pm 84.6$ & $19.15 \pm 0.09$ & $<1$ & $30.2 \pm 0.15$ & $126.3 \pm 0.63$ & $595.2 \pm 2.97$ & $100 \pm 0.50$ & $47 \pm 0.28$ & $118.5 \pm 0.59$ & $1339 \pm 6.69$ \\
\hline SD05 & $<1$ & $31.95 \pm 0.15$ & $133.6 \pm 0.66$ & $185.7 \pm 0.92$ & $91.4 \pm 0.45$ & $761.0 \pm 3.80$ & $101.8 \pm 0.51$ & $10 \pm 0.05$ & $401.7 \pm 2.05$ & $2517 \pm 12.58$ \\
\hline SD06 & $474.7 \pm 2.37$ & $31.95 \pm 0.15$ & $39.29 \pm 0.19$ & $83.6 \pm 0.41$ & $210.3 \pm 1.21$ & $532.7 \pm 2.66$ & $50.54 \pm 0.25$ & $8 \pm 0.04$ & $321.4 \pm 1.60$ & $962 \pm 4.81$ \\
\hline SD07 & $2856 \pm 14.28$ & $21.53 \pm 0.11$ & $<1$ & $57.3 \pm 0.28$ & $99.7 \pm 0.49$ & $438.1 \pm 2.19$ & $106 \pm 0.53$ & $16 \pm 0.08$ & $176.1 \pm 0.88$ & $1886 \pm 9.43$ \\
\hline SD08 & $3094 \pm 15.47$ & $21.07 \pm 0.10$ & $<1$ & $49.9 \pm 0.25$ & $137.9 \pm 0.68$ & $603.5 \pm 3.01$ & $107 \pm 0.53$ & $33 \pm 0.16$ & $220.0 \pm 1.10$ & $2788 \pm 13.94$ \\
\hline SD09 & $209.7 \pm 1.05$ & $23.97 \pm 0.12$ & $<1$ & $120.7 \pm 0.60$ & $181.8 \pm 0.90$ & $813.8 \pm 4.06$ & $55.72 \pm 0.27$ & $10 \pm 0.05$ & $273.2 \pm 1.36$ & $1890 \pm 9.45$ \\
\hline SD10 & $447.4 \pm 2.24$ & $<1$ & $<1$ & $185.7 \pm 0.93$ & $181.8 \pm 0.90$ & $292.6 \pm 1.46$ & $64.69 \pm 0.32$ & $36 \pm 0.18$ & $192.8 \pm 0.96$ & $4095 \pm 20.47$ \\
\hline SD11 & $251.7 \pm 1.21$ & $47.93 \pm 0.24$ & $47.15 \pm 0.23$ & $148.5 \pm 0.74$ & $189.4 \pm 0.94$ & $786.4 \pm 3.93$ & $76.41 \pm 0.38$ & $27 \pm 0.14$ & $249.1 \pm 1.24$ & $2913 \pm 14.56$ \\
\hline SD12 & $321.6 \pm 1.61$ & $15.98 \pm 0.08$ & $<1$ & $371.3 \pm 1.85$ & $212.1 \pm 1.06$ & $640.1 \pm 3.20$ & $57.4 \pm 0.28$ & $22 \pm 0.11$ & $257.1 \pm 1.28$ & $2205 \pm 11.02$ \\
\hline SD13 & $2414 \pm 12.07$ & $21.05 \pm 0.10$ & $<1$ & $49.9 \pm 0.25$ & $114.1 \pm 0.57$ & $652.2 \pm 3.26$ & $95 \pm 0.47$ & $24 \pm 0.12$ & $219.8 \pm 1.09$ & $2962 \pm 14.81$ \\
\hline SD14 & $3066 \pm 15.33$ & $20.83 \pm 0.10$ & $<1$ & $49.4 \pm 0.25$ & $136.3 \pm 0.68$ & $596.7 \pm 3.98$ & $84 \pm 0.42$ & $31 \pm 0.15$ & $217.5 \pm 1.08$ & $2755 \pm 13.77$ \\
\hline LR & $243.6 \pm 1.21$ & $5.49 \pm 0.02$ & $5.66 \pm 0.03$ & $91.3 \pm 0.45$ & $138.8 \pm 0.69$ & $783.8 \pm 3.41$ & $52.26 \pm 0.26$ & $18.59 \pm 0.09$ & $139.8 \pm 0.69$ & $1571 \pm 7.85$ \\
\hline
\end{tabular}

$\mathrm{SD}=$ Sediment.

Source: research data. LR - Local Rock. Geochemistry data reported by (Valeton et al., 1997). 
The elemental concentrations found in potential sources of contamination-PSC (fertilizers, wastes and caldasite) are shown in Table 2.

Table 2. Potential sources of contamination concentrations.

\begin{tabular}{|c|c|c|c|c|c|c|c|c|c|c|c|}
\hline & \multirow[b]{2}{*}{ Samples } & \multicolumn{10}{|c|}{ ELEMENTS (\%) } \\
\hline & & $\mathrm{Al}$ & $\mathrm{Ca}$ & $\mathrm{Fe}$ & $\mathrm{Mg}$ & $\mathrm{Mn}$ & $\mathrm{Na}$ & $\mathrm{K}$ & $\mathrm{P}$ & $\mathrm{Si}$ & $\mathrm{Ti}$ \\
\hline \multirow{4}{*}{$\mathrm{BF} 4$} & RM-03 & $15.56 \pm 0.07$ & $0.03 \pm 1 \times 10^{-4}$ & $4.55 \pm 0.02$ & $0.07 \pm 3 \times 10^{-4}$ & $0.19 \pm 9 \times 10^{-4}$ & $0.07 \pm 3 \times 10^{-4}$ & $10.04 \pm 0.05$ & $0.11 \pm 5 \times 10^{-4}$ & $23.00 \pm 0.11$ & 0.44 \\
\hline & RM-04 & $14.71 \pm 0.07$ & $0.09 \pm 4 \times 10^{-4}$ & $3.92 \pm 0.02$ & $0.06 \pm 3 \times 10^{-4}$ & $0.15 \pm 7 \times 10^{-4}$ & $0.10 \pm 5 \times 10^{-4}$ & $10.63 \pm 0.05$ & $0.11 \pm 5 \times 10^{-4}$ & $23.75 \pm 0.12$ & 0.44 \\
\hline & RM-01 & $16.37 \pm 0.08$ & $0.29 \pm 1 \times 10^{-3}$ & $5.15 \pm 0.02$ & $0.11 \pm 5 \times 10^{-4}$ & $0.08 \pm 4 \times 10^{-4}$ & $0.22 \pm 1 \times 10^{-3}$ & $3.56 \pm 0.01$ & $0.07 \pm 3 \times 10^{-4}$ & $17.78 \pm 0.08$ & 0.49 \\
\hline & BF4-Mean & $15.55 \pm 0.07$ & $0.14 \pm 7 \times 10^{-4}$ & $4.54 \pm 0.07$ & $0.08 \pm 4 \times 10^{-4}$ & $0.14 \pm 7 \times 10^{-4}$ & $0.13 \pm 6 \times 10^{-4}$ & $8.08 \pm 0.04$ & $0.10 \pm 5 \times 10^{-4}$ & $21.51 \pm 0.11$ & 0.46 \\
\hline \multirow{4}{*}{$\mathrm{BR}$} & RM-05 & $13.97 \pm 0.07$ & $0.04 \pm 2 \times 10^{-4}$ & $1.47 \pm 7 \times 10^{-3}$ & $0.04 \pm 2 \times 10^{-4}$ & $0.01 \pm 5 \times 10^{-5}$ & $0.06 \pm 3 \times 10^{-4}$ & $12.45 \pm 0.06$ & $0.08 \pm 4 \times 10^{-4}$ & $25.15 \pm 0.12$ & 0.50 \\
\hline & RM-06 & $14.55 \pm 0.07$ & $0.02 \pm 1 \times 10^{-4}$ & $2.59 \pm 0.01$ & $0.04 \pm 2 \times 10^{-4}$ & $0.01 \pm 5 \times 10^{-5}$ & $0.06 \pm 3 \times 10^{-4}$ & $11.54 \pm 0.05$ & $0.07 \pm 3 \times 10^{-4}$ & $23.98 \pm 0.12$ & 0.66 \\
\hline & RM-02 & $13.64 \pm 0.06$ & $1.66 \pm 8 \times 10^{-3}$ & $5.22 \pm 0.02$ & $0.27 \pm 1 \times 10^{-3}$ & $5.35 \pm 0.02$ & $0.21 \pm 1 \times 10^{-3}$ & $2.19 \pm 0.01$ & $0.10 \pm 5 \times 10^{-4}$ & $10.52 \pm 0.05$ & 2.71 \\
\hline & BR-Mean & $14.06 \pm 0.07$ & $0.57 \pm 3 \times 10^{-4}$ & $3.09 \pm 0.01$ & $0.12 \pm 6 \times 10^{-4}$ & $1.79 \pm 0.01$ & $0.11 \pm 5 \times 10^{-4}$ & $8.73 \pm 0.04$ & $0.09 \pm 4 \times 10^{-4}$ & $19.88 \pm 0.10$ & 1.29 \\
\hline \multirow{8}{*}{ FT } & FT-01 & $0.21 \pm 1 \times 10^{-4}$ & $31.73 \pm 0.15$ & $2.03 \pm 0.01$ & $0.23 \pm 1 \times 10^{-3}$ & $0.13 \pm 6 \times 10^{-4}$ & $0.13 \pm 6 \times 10^{-4}$ & $0.70 \pm 3 \times 10^{-3}$ & $7.64 \pm 0.03$ & $0.65 \pm 3 \times 10^{-4}$ & $0.30 \pm 1 \times 10^{-4}$ \\
\hline & FT-02 & $<0.1$ & $0.31 \pm 1 \times 10^{-3}$ & $0.02 \pm 1 \times 10^{-5}$ & $0.00 \pm 1 \times 10^{-5}$ & $0.00 \pm 2 \times 10^{-5}$ & $0.02 \pm 1 \times 10^{-4}$ & $0.13 \pm 6 \times 10^{-4}$ & $0.88 \pm 4 \times 10^{-3}$ & $0.35 \pm 1 \times 10^{-4}$ & $<0.01$ \\
\hline & FT-03 & $<0.1$ & $9.91 \pm 0.49$ & $1.56 \pm 7 \times 10^{-3}$ & $0.00 \pm 1 \times 10^{-5}$ & $0.07 \pm 3 \times 10^{-5}$ & $4.01 \pm 0.02$ & $24.65 \pm 0.12$ & $2.40 \pm 0.01$ & $0.41 \pm 2 \times 10^{-4}$ & $<0.01$ \\
\hline & FT-04 & $0.26 \pm 1 \times 10^{-4}$ & $24.01 \pm 0.12$ & $1.54 \pm 7 \times 10^{-3}$ & $0.38 \pm 1 \times 10^{-3}$ & $0.09 \pm 4 \times 10^{-5}$ & $0.38 \pm 1 \times 10^{-3}$ & $7.80 \pm 0.03$ & $6.20 \pm 0.03$ & $0.79 \pm 4 \times 10^{-4}$ & $0.22 \pm 1 \times 10^{-4}$ \\
\hline & FT-05 & $1.33 \pm 0,06$ & $51.82 \pm 0.25$ & $0.63 \pm 3 \times 10^{-3}$ & $9.17 \pm 0.04$ & $0.15 \pm 7 \times 10^{-4}$ & $0.02 \pm 1 \times 10^{-4}$ & $0.37 \pm 1 \times 10^{-3}$ & $0.10 \pm 5 \times 10^{-4}$ & $3.01 \pm 0.01$ & $0.07 \pm 3 \times 10^{-5}$ \\
\hline & FT-06 & $<0.1$ & $25.03 \pm 0.12$ & $0.23 \pm 1 \times 10^{-3}$ & $1.50 \pm 7 \times 10^{-3}$ & $0.00 \pm 2 \times 10^{-5}$ & $4.89 \pm 0.02$ & $24.18 \pm 0.12$ & $0.03 \pm 1 \times 10^{-4}$ & $0.56 \pm 3 \times 10^{-4}$ & $<0.01$ \\
\hline & FT-07 & $3.97 \pm 0,02$ & $18.15 \pm 0.09$ & $5.95 \pm 0.02$ & $6.87 \pm 0.03$ & $0.77 \pm 3 \times 10^{-3}$ & $1.19 \pm 5 \times 10^{-3}$ & $1.58 \pm 7 \times 10^{-3}$ & $6.15 \pm 0.03$ & $10.24 \pm 0.05$ & $0.35 \pm 1 \times 10^{-4}$ \\
\hline & FT-Mean & $1.44 \pm 7 \times 10^{-3}$ & $23.00 \pm 0.12$ & $1.71 \pm 8 \times 10^{-3}$ & $2.59 \pm 0.01$ & $0.18 \pm 9 \times 10^{-4}$ & $1.52 \pm 6 \times 10^{-3}$ & $8.49 \pm 0.04$ & $3.34 \pm 0.01$ & $2.29 \pm 0.01$ & $0.24 \pm 1 \times 10^{-4}$ \\
\hline \multirow[t]{3}{*}{$\mathrm{CT}$} & $\mathrm{CT}$ & $7.41 \pm 0.03$ & $0.09 \pm 4 \times 10^{-4}$ & $27.98 \pm 0.13$ & $0.03 \pm 1 \times 10^{-4}$ & $3.18 \pm 0.01$ & $0.02 \pm 1 \times 10^{-4}$ & $1.99 \pm 0,01$ & $0.65 \pm 3 \times 10^{-4}$ & $6.26 \pm 0.03$ & $0.31 \pm 6 \times 10^{-4}$ \\
\hline & & \multicolumn{10}{|c|}{ ELEMENTS $\left(\mathrm{mg} \mathrm{kg}^{-1}\right)$} \\
\hline & Samples & $\mathrm{Ba}$ & $\mathrm{Cu}$ & $\mathrm{Ni}$ & $\mathrm{Pb}$ & $\mathrm{Rb}$ & $\mathrm{Sr}$ & Th & $\mathrm{U}$ & $\mathrm{Zn}$ & $\mathrm{Zr}$ \\
\hline \multirow{4}{*}{$\mathrm{BF} 4$} & RM-03 & $895.7 \pm 4.42$ & $<1$ & $<1$ & $380.6 \pm 1.90$ & $365.8 \pm 1.32$ & $710.3 \pm 3.55$ & $99.8 \pm 0.49$ & $121 \pm 0.65$ & $136.6 \pm 0.68$ & $2073 \pm 10.36$ \\
\hline & RM-04 & $1075 \pm 5.32$ & $<1$ & $<1$ & $157.8 \pm 7.89$ & $384.1 \pm 1.92$ & $676.5 \pm 3.38$ & $103.4 \pm 0.51$ & $169 \pm 0.84$ & $120.5 \pm 0.60$ & $2443 \pm 12.21$ \\
\hline & RM-01 & $1373 \pm 6.31$ & $18.38 \pm 0,09$ & $<1$ & $74.5 \pm 0.37$ & $129.6 \pm 0.64$ & $380.2 \pm 1.90$ & $430.0 \pm 2.10$ & $480 \pm 2.40$ & $928.7 \pm 4.64$ & $1988 \pm 9.94$ \\
\hline & BF4-Mean & $1115 \pm 5.57$ & $<1$ & - & $204.3 \pm 1.02$ & $293.1 \pm 1.46$ & $589.0 \pm 2.94$ & $211.1 \pm 1.05$ & $256.7 \pm 1.28$ & $395.3 \pm 1.97$ & $2168 \pm 10.84$ \\
\hline \multirow{4}{*}{$\mathrm{BR}$} & RM-05 & $985.2 \pm 4.92$ & $<1$ & $<1$ & $176.4 \pm 0.88$ & $457.2 \pm 2.26$ & $456.6 \pm 2.28$ & $39.4 \pm 0.19$ & $81 \pm 0.40$ & $24.1 \pm 0.12$ & $2591 \pm 12.95$ \\
\hline & RM-06 & $1344 \pm 6.72$ & $<1$ & $<1$ & $232.1 \pm 1.16$ & $457.2 \pm 2.26$ & $431.3 \pm 2.15$ & $56.7 \pm 0.28$ & $95 \pm 0.47$ & $32.1 \pm 0.16$ & $3924 \pm 14.62$ \\
\hline & RM-02 & $3633 \pm 13.16$ & $22.10 \pm 0.11$ & $<1$ & $16.1 \pm 0.08$ & $94.1 \pm 0.47$ & $4807.9 \pm 24.03$ & $277.0 \pm 1.38$ & $100 \pm 0.50$ & $1052 \pm 5.26$ & $971.1 \pm 4.85$ \\
\hline & BR-Mean & $1987 \pm 9.93$ & $22.10 \pm 0.11$ & - & $141.5 \pm 0.71$ & $336.2 \pm 1.68$ & $1898.6 \pm 9.49$ & $124.3 \pm 0.62$ & $92 \pm 0.46$ & $369.4 \pm 1.84$ & $2495 \pm 12.47$ \\
\hline \multirow{8}{*}{ FT } & FT-01 & $4210 \pm 21.05$ & $143.8 \pm 0.71$ & $102.2 \pm 0.51$ & $<1$ & $<1$ & $11838 \pm 59.19$ & $65.8 \pm 0.32$ & $72 \pm 0.36$ & $257.1 \pm 1.28$ & $37.0 \pm 0.16$ \\
\hline & FT-02 & $<1$ & $79.89 \pm 0.39$ & $<1$ & $<1$ & $<1$ & $42.3 \pm 0.21$ & $0.3 \pm 1 \times 10^{-3}$ & $1 \pm 5 \times 10^{-3}$ & $40.2 \pm 0.20$ & $<1$ \\
\hline & FT-03 & $<1$ & $159.8 \pm 0.79$ & $<1$ & $<1$ & $<1$ & $2537 \pm 12.68$ & $53.6 \pm 0.26$ & $12 \pm 0.06$ & $200.9 \pm 1.01$ & $<1$ \\
\hline & FT-04 & $42010 \pm 210.05$ & $95.86 \pm 0.47$ & $70.72 \pm 0.35$ & $120.7 \pm 0.60$ & $<1$ & $5835 \pm 29.16$ & $48.8 \pm 0.24$ & $48 \pm 0.24$ & $160.7 \pm 0.80$ & $266.5 \pm 1.33$ \\
\hline & FT-05 & $<1$ & $<1$ & $78.58 \pm 0.39$ & $<1$ & $<1$ & $2368 \pm 11.84$ & $0.3 \pm 1 \times 10^{-3}$ & $2 \pm 0.01$ & $112.5 \pm 0.56$ & $<1$ \\
\hline & FT-06 & $<1$ & $207.7 \pm 1.03$ & $<1$ & $<1$ & $<1$ & $338.2 \pm 1.69$ & $33.0 \pm 0.16$ & $16 \pm 0.08$ & $40.2 \pm 0.02$ & $<1$ \\
\hline & FT-07 & $26870 \pm 134.35$ & $591.2 \pm 2.95$ & $722.9 \pm 3.61$ & $102.1 \pm 0.60$ & $45.7 \pm 0.22$ & $5666 \pm 28.33$ & $1.3 \pm 0.06$ & $1 \pm 5 \times 10^{-3}$ & $5865 \pm 29.32$ & $177.7 \pm 0.88$ \\
\hline & FT-Mean & $11763 \pm 56.81$ & $213.0 \pm 1.06$ & $243.6 \pm 1.21$ & $111.4 \pm 0.55$ & $45.7 \pm 0.22$ & $4089 \pm 20.44$ & $29.0 \pm 0.14$ & $21.64 \pm 0.11$ & $953.8 \pm 4.76$ & $160.4 \pm 0.80$ \\
\hline CT & $\mathrm{CT}$ & $1701.7 \pm 6.00$ & $<1$ & $<1$ & $529.1 \pm 2.14$ & $<1$ & $2114.0 \pm 10.57$ & $611.1 \pm 30.55$ & 1200 & $498.1 \pm 2.49$ & $139921 \pm 699.60$ \\
\hline
\end{tabular}

FT=fertilizers; BF4-mean= mean of samples RM-03, RM-04 and RM-01; BR-mean= mean of samples RM-05, RM-06 and RM-02; CT=Caldasite.

Source: research data.

Rev. Ambient. Água vol. 14 n. 5, e2397 - Taubaté 2019 
After calculating the enrichment factor (EF), the nine elements that obtained FE $>3$ in at least one (1) sediment sample are represented in Table 3: $\mathrm{Ba}, \mathrm{Cu}, \mathrm{Mg}, \mathrm{Mn}, \mathrm{Ni}, \mathrm{P}, \mathrm{Pb}$ and $\mathrm{U}$. The EF values calculated from the mean concentration of each PSC are shown in Table 3.

Table 3. Enrichment Factor for elements with at least 1 sediment sample with $E F>3$. No colour $(\mathrm{EF} \leq 3)$, green $(3<\mathrm{EF} \leq 5)$, blue $(5<\mathrm{EF} \leq 10)$, orange $(10<\mathrm{EF} \leq 25)$, red $(\mathrm{EF}>50)$.

\begin{tabular}{ccccccccccccc}
\hline & $\mathrm{Ba}$ & $\mathrm{Cu}$ & $\mathrm{Mg}$ & $\mathrm{Mn}$ & $\mathrm{Ni}$ & $\mathrm{P}$ & $\mathrm{Pb}$ & $\mathrm{Th}$ & $\mathrm{U}$ & Total & WR & WE \\
\hline SD01 & - & - & 0.68 & 0.35 & - & 10.72 & 1.72 & 1.13 & 0.48 & 1 & 10.72 & $\mathrm{P}$ \\
SD02 & 1.50 & 2.55 & 0.73 & 2.11 & - & 5.89 & 4.97 & 1.85 & 2.98 & 2 & 5.89 & $\mathrm{P}$ \\
SD03 & 0.73 & - & 0.65 & 2.97 & - & 3.40 & 1.60 & 1.61 & 8.91 & 1 & 3.40 & $\mathrm{U}$ \\
SD04 & 12.40 & 3.26 & 1.60 & 1.02 & - & 4.38 & 0.59 & 3.42 & 4.51 & 4 & 12.40 & $\mathrm{Ba}$ \\
SD05 & - & 4.68 & 1.10 & 0.80 & - & 3.27 & 0.92 & 0.88 & 0.24 & 2 & 4.68 & $\mathrm{Cu}$ \\
SD06 & 1.40 & 4.66 & 0.85 & 0.52 & 5.56 & 5.45 & 0.66 & 0.70 & 0.31 & 3 & 5.56 & $\mathrm{Ni}$ \\
SD07 & 8.26 & 3.78 & 1.36 & 1.04 & - & 1.76 & 0.44 & 1.43 & 0.61 & 2 & 8.26 & $\mathrm{Ba}$ \\
SD08 & 9.52 & 3.77 & 5.44 & 1.07 & - & 2.01 & 0.41 & 1.53 & 1.33 & 3 & 9.52 & $\mathrm{Ba}$ \\
SD09 & 0.61 & - & 0.83 & 3.09 & - & 3.05 & 0.94 & 0.76 & 0.38 & 2 & 3.09 & $\mathrm{Mn}$ \\
SD10 & 1.28 & - & 0.87 & 1.70 & - & 2.41 & 1.42 & 0.87 & 1.35 & 0 & 2.41 & $\mathrm{P}$ \\
SD11 & 0.82 & - & 0.98 & 4.96 & - & 3.80 & 1.28 & 1.15 & 1.15 & 2 & 4.96 & $\mathrm{Mn}$ \\
SD12 & 0.78 & 2.39 & 0.77 & 2.27 & - & 2.84 & 2.40 & 0.65 & 0.70 & 0 & 2.84 & $\mathrm{P}$ \\
SD13 & 7.43 & 3.77 & 1.24 & 1.07 & - & 2.01 & 0.41 & 1.36 & 0.97 & 2 & 7.43 & $\mathrm{Ba}$ \\
SD14 & 9.53 & 3.75 & 2.30 & 1.07 & - & 2.01 & 0.41 & 1.22 & 1.26 & 2 & 9.53 & $\mathrm{Ba}$ \\
EF>3 (SD) & 5 & 7 & 1 & 2 & 1 & 8 & 1 & 1 & 2 & & & \\
\hline BF4-Mean & 3.45 & 2.52 & 0.69 & 1.01 & - & 2.97 & 1.69 & 3.04 & 10.41 & 2 & 3.45 & $\mathrm{U}$ \\
BR-Mean & 9.03 & 4.46 & 1.52 & 18.91 & - & 3.93 & 1.72 & 2.64 & 5.48 & 4 & 18.91 & $\mathrm{Mn}$ \\
FT-Mean & 96.62 & 77.67 & 59.41 & 3.44 & 86.18 & 263.60 & 2.44 & 1.11 & 2.33 & 6 & 263.60 & $\mathrm{P}$ \\
CT & 0.85 & - & 0.04 & 3.71 & - & 3.14 & 0.71 & 1.43 & 7.89 & 2 & 3.71 & $\mathrm{U}$ \\
EF>3 (souces) & 3 & 2 & 1 & 3 & 1 & 3 & 0 & 1 & 3 & & & \\
\hline
\end{tabular}

$\mathrm{HE}=$ Highest Enrichment; $\mathrm{EE}=$ Most Enriched Element; $\mathrm{SD}=$ sediment; $\mathrm{CT}=$ caldasite; $\mathrm{FT}=$ fertilizers; $\mathrm{BR}$ and $\mathrm{BF} 4=$ wastes.

Source: research data.

The distribution of EF for sediments and PSC is shown in Figure 2. Phosphorus is an important macronutrient for plants and is very present in the analyzed fertilizers (Table 3 and Figure 2), with an extremely severe enrichment, while only moderately enriched in BR and CT. It is the element with the highest occurrence of results (8) with EF> 3 in the fluvial sediments: moderately enriched (SD03, SD04, SD05, SD09 and SD11), moderately severe enrichment (SD02 and SD06) to severe enrichment (SD01). Phosphorus is the most enriched element (Figure 3) in samples SD01, SD02, SD06, SD09, SD10 and SD12. The samples SD01, SD02, SD09 and SD10 are located upstream of the CUM and the caldasite ore. In these samples, fertilizers are probably the source of phosphorus enrichment.

In the SD06 sample, phosphorus and nickel (present only in SD06) are the most enriched elements. Nickel is a micronutrient for plants, and its occurrence in PSC is restricted to the analyzed fertilizers (Table 3 and Figure 2). As will be seen later, this sample is moderately enriched in copper. This $\mathrm{P}-\mathrm{Ni}-\mathrm{Cu}$ association has a severe enrichment in the evaluated fertilizers, it strengthens them as the cause of the P enrichment verified in the SD06 sample. The SD12 sample occurs in the agricultural area downstream of the CT. In this sample, the phosphorus must be related to fertilizers, but the contribution of the CT cannot be ruled out.

Copper is a micronutrient for plants; therefore it occurs in fertilizers with extremely severe enrichment, while it is moderately enriched in BR. In the sediments it is moderately enriched in 7 samples: SD04, SD05, SD06, SD07, SD08, SD13 and SD14. This copper enrichment, although moderate, is a strong indication of fertilizer input. $\mathrm{Cu}$ occurs as the most enriched element in sample SD05 (Figure 3). 


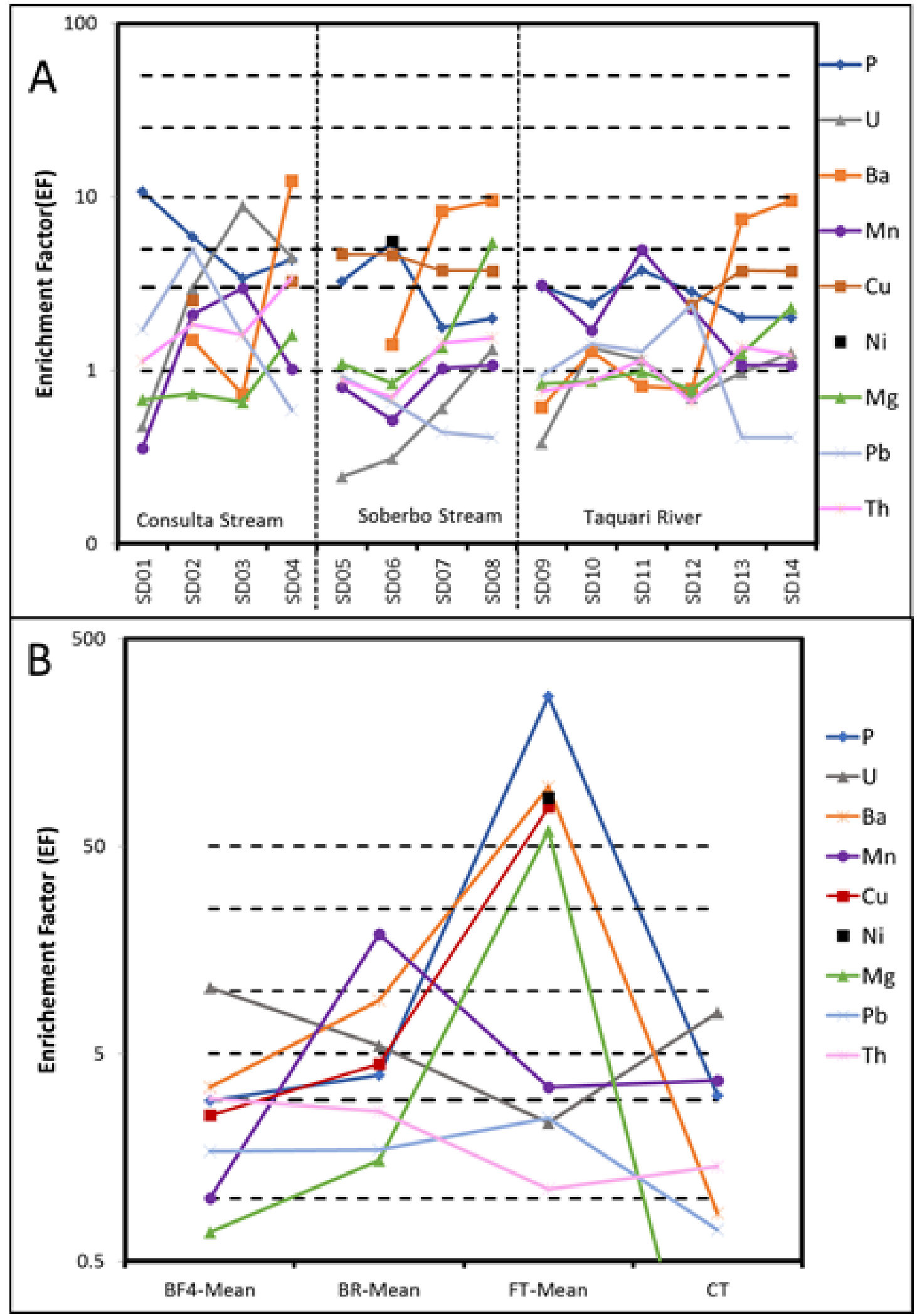

Figure 2. Distribution of EF values in A) sediments and in B) sources of contamination. Source: research data.

Barium is moderately enriched in BR tailings, but presents extremely severe enrichment in the analyzed fertilizers. In BR, the enrichment in Ba certainly arises from the addition of $\mathrm{BaCl}_{2}$ to treat $\mathrm{BR}$ effluents. $\mathrm{Ba}$ is not a plant nutrient, but is present associated with calcium 
and phosphorus in fertilizers. In the evaluated sediments, barium presents moderate enrichment (SD07, SD08, SD13 and SD14) to severe enrichment (SD04). In SD04 sample certainly the fertilizer application is the cause of the enrichment. In the other samples, the enrichment must come from fertilizers and/or from BR effluent discharges, since all the samples are in the drainage line downstream of this dam.

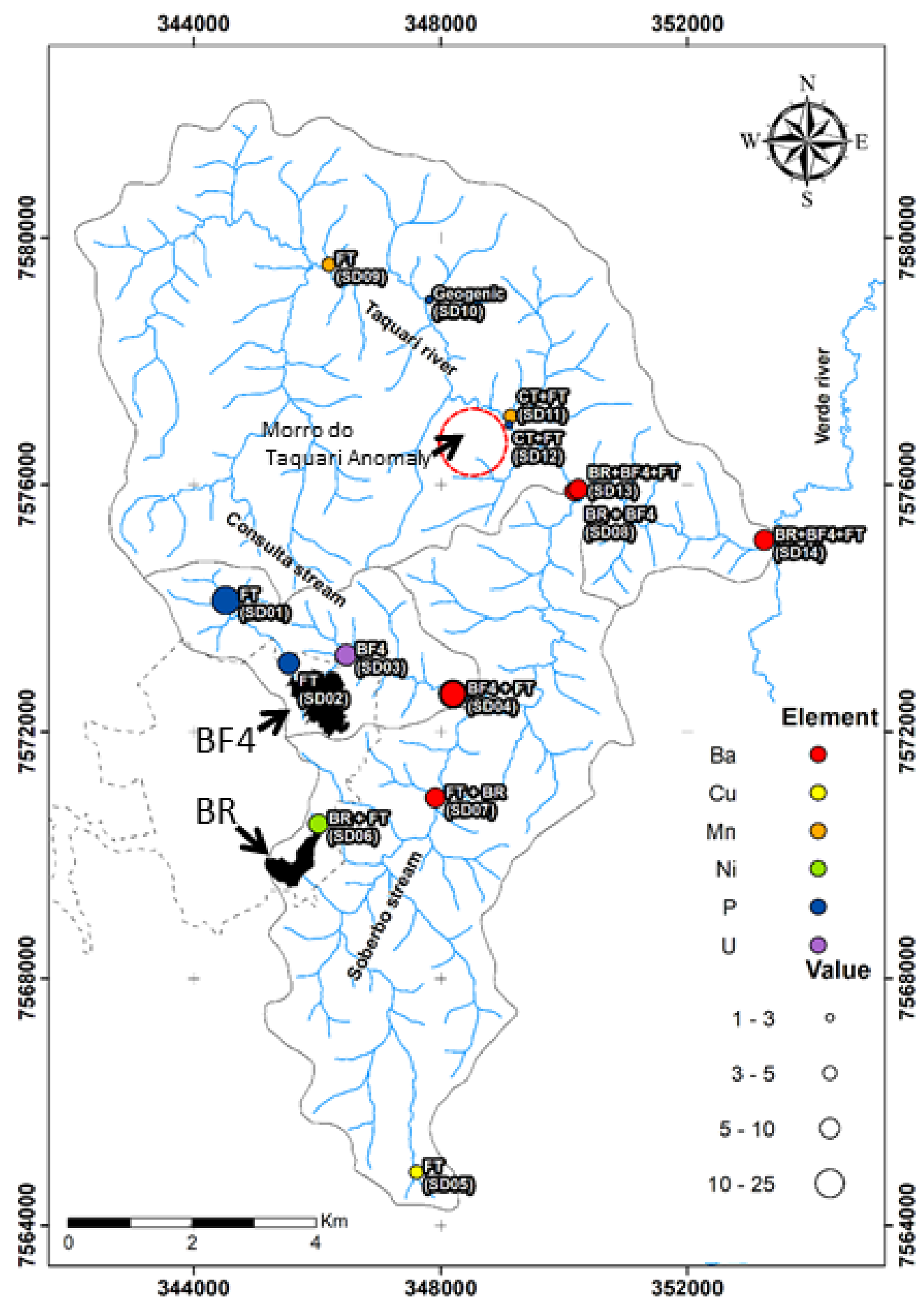

Figure 3. Distribution of the most enriched elements in each of the sediment samples and an indication of the likely source (s) for enrichment.

Source: research data.

Uranium has EF ranging from moderately severe enrichment ( $\mathrm{CT}$ and $\mathrm{BR}$ ) to severe enrichment (BF4). In the sediments it is enriched in samples SD04 (moderately enriched) and 
SD03 (moderately severe enrichment), being the element more enriched in the latter. BF4 effluents are certainly responsible for this uranium enrichment. In the potential sources of contamination Mn has a moderately enriched (CT and FT) to moderately severe enrichment (BR). In the mineral treatment process crushed pyrolusite $\left(\mathrm{MnO}_{2}\right)$ was added to act as a uranium ore oxidizing agent (Golder, 2012). This source of exogenous Mn must be the main cause for the enrichment in Mn in the wastes deposited in BR. Mn is a micronutrient so it is found in the analyzed fertilizers. In the fluvial sediments Mn occurs moderately enriched in samples SD09 and SD11, in which it is the most enriched element. In sample SD09, located in an area with exclusively agricultural occupation, the presence of $\mathrm{Mn}$ (together with $\mathrm{P}$ ) indicates the fertilizers as cause of the enrichment. In the SD11 sample, Mn may come from the fertilizers and/or from the caldasite.

Magnesium (macronutrient for plants) occurs only in fertilizers with EF> 50 (extremely severe enrichment). In the sediments it is enriched only in sample SD08 (moderately severe enrichment), probably due to the application of fertilizers. The $\mathrm{Pb}$ is not enriched in any of the PSC, whereas in the sediments it occurs moderately enriched in SD02 sample. It is difficult to associate this enrichment with any source, but since this sample was collected upstream of BF4, $\mathrm{Pb}$ must come from fertilizers. Thorium is enriched (moderately) only in BF4, which should be the thorium source for sample SD04.

\section{CONCLUSION}

The results obtained in this study provide an overview of the concentrations of the selected chemical elements in sediments of water bodies in the Taquari River Basin. These results indicate relevance for this region, considering the large presence of areas with fertilizer application, mining areas and natural anomaly. The application of the enrichment factor showed that the fluvial sediments present moderate to severe enrichment in $\mathrm{P}, \mathrm{Cu}, \mathrm{Ba}, \mathrm{U}, \mathrm{Mn}, \mathrm{Ni}, \mathrm{Mg}$, $\mathrm{Pb}$ and $\mathrm{Th}$, indicating the predominant influences of anthropogenic material and natural anomalies.

\section{ACKNOWLEDGEMENTS}

The authors thank FAPEMIG for financial support, and the Nuclear Industries of Brazil (INB) for their technical and operational cooperation.

\section{REFERENCES}

AGBENIN, J. O. The distribution and dynamics of chromium and nickel in cultivated and uncultivated semi-arid soils from Nigeria. Science of Total Environment, v. 300, n. 13, p.189-199, 2002. https://doi.org/10.1016/S0048-9697(02)00231-0

CARVALHO FILHO, C. A. de; MOREIRA R. M.; GUIMARÃES B. F.; FERREIRA V. V. M.; AULER L. M. L. A.; PALMIERI H. E. L.; OLIVEIRA A. F. G.; DUTRA P. H. Hydrochemical assessment of surface water in watersheds near the Uranium Mining and Milling Facilities of Caldas. Brazil. Environmental Earth Sciences, v. 75, n. 3, Article 187, 2016. https://doi.org/10.1007/s12665-015-5070-7

CARVALHO FILHO, C. A. de; MOREIRA R. M.; BRANCO O. E. A.; DUTRA P. H.; SANTOS, E.A.; MOURA, I. F. S.; FLEMING, P. M.; PALMIERI, H. E. L. Combined hydrochemical, isotopic, and multivariate statistics techniques to assess the effects of discharges from a uranium mine on water quality in neighboring streams. Environmental Earth Sciences, v. 76, 2017. https://doi.org/10.1007/s12665-017-7165-9 
CHEN, C. W.; KAO, C. M.; CHEN, C. F.; DONG, C. D. Distribution and accumulation of heavy metals in the sediments of Kaohsiung Harbor, Taiwan. Chemosphere, v. 66, n. 8, p. 1431-1440, 2007. https://doi.org/10.1016/j.chemosphere.2006.09.030

DIN, Z. B. Use of Aluminum to Normalize Heavy-Metal Data from Estuarine and Coastal Sediments of Straits of Melaka. Marine Pollution Bulletin, v. 24, n. 10, p. 484-491, 1992. https://doi.org/10.1016/0025-326X(92)90472-I

EMBRAPA. Agrotóxicos e Ambiente. EMBRAPA, Brasília, 2004. 400 p.

FERNANDES, H. M.; VEIGA L. H. S.; FRANKLIN, M. R.; PRADO, V. C. S.; TADDEI, J. F. Environmental impact assessment of uranium and milling facilities: a study case at Poços de Caldas uranium mining and milling site, Brazil. Journal of Geochemical Exploration, v. 52, p.161-173, 1995. https://doi.org/10.1016/0375-6742(94)00043-B

FERNANDES, H. M.; FRANKLIN, M. R.; VEIGA L. H. S. Acid rock drainage and radiological environmental impacts: a study case of the Uranium mining and milling facilities at Poços de Caldas. Waste Management v. 18, n. 1, p. 169-181, 1998. https://doi.org/10.1016/S0956-053X(98)00019-1

FREITAS, E. V. S.; NASCIMENTO C. W. A.; GOUlART, D. F.; SILVA, J. P.S. Disponibilidade de cádmio e chumbo para milho em solo adubado com fertilizantes fosfatados. Revista Brasileira de Ciências do Solo, v. 33, n. 6, p. 1899-1907, 2009. http://dx.doi.org/10.1590/S0100-06832009000600039

GOLDER ASSOCIATES BRAZIL CONSULTING AND PROJECTS LTD. Plan for the Recovery of Degraded areas - INB UTM Caldas. Technical Report No. RT-006_099515-3023_01-j. Brazil, 2012.

JACOMINO, V. M. F.; OLIVEIRA, K. A. P.; TADDEI, M. H. T.; SIQUEIRA, M. C.; CARNEIRO, M. E. D. P.; NASCIMENTO, M. R. L.; SILVA, D. F.; MELLO, J. W. V. Radionuclides and heavy metal contents in phosphogypsum samples in comparison to cerrado soils. Revista Brasileira de Ciências do Solo, v. 33, p. 1481-1488, 2009. http://dx.doi.org/10.1590/S0100-06832009000500038

MARNIKA, E., CHRISTODOULOU, E., XENIDIS, A. Sustainable development indicators for mining sites in protected areas: tool development, ranking and scoring of potential environmental impacts and assessment of management scenarios. Journal of Cleaner Production v. 101, p. 59-70, 2015. http://dx.doi.org/10.1016/j.jclepro.2015.03.098 09596526

MENEZES, M. A. B. C.; JACIMOVIC, R. Optimized k0-instrumental Neutron Activation Method using the TRIGA MARK I IPR-R1 Reactor at CDTN/CNEN, Belo Horizonte, Brazil. Nuclear Instruments \& Methods in Physics Research, v. 564, p. 707-715, 2006.

REIS, A.; PARKER, A.; ALENCOÃO, A. Avaliação da qualidade de sedimentos em rios de Montanha: Um caso de estudo no norte de Portugal. Associação Portuguesa dos Recursos Hídricos, v. 31, n. 1, p. 87-97, 2010.

SAUEIA, C. H. R.; MAZZILLI, B. P. Distribution of natural radionuclides in the production and use of phosphate fertilizers in Brazil. Journal of Environmental Radioactivity, v. 89, n. 3, p. 229-239, 2006. https://dx.doi.org/10.1016/j.jenvrad.2006.05.009

SAVCI, S. Investigation of the Effect of Chemical Fertilizers on Environment. APCBEE Procedia, n. 1, p. 287-292, 2012. https://doi.org/10.1016/j.apcbee.2012.03.047 
SCHROPP, S. J.; LEWIS, F. G.; WINDOM, H. L.; RYAN, J. D.; CALDER, F. D.; BURNEY, L. C. Interpretation of metal concentrations in estuarine sediments of Florida using aluminum as a reference element. Estuaries, v. 13, n. 3, p. 227-235, 1990. https://doi.org/10.2307/1351913

SCHORSCHER, H. D.; SHEA, M. E. The Regional Geology of the Poços de Caldas Alkaline Complex: mineralogy and geochemistry of selected nepheline syenites and phonolites. Journal of Geochemical Exploration, v. 45, n. 1-3, p. 25-51, 1992. https://doi.org/10.1016/0375-6742(92)90121-N

SELVARAJ, K.; MOHAN, V. R.; SZEFER, P. Evaluation of metal contamination in coastal sediments of the Bay of Bengal, India: geochemical and statistical approaches. Marine $\begin{array}{llllll}\text { Pollution Bulletin } & \text { v. } & \text { 49, } & \text { p. }\end{array}$ https://doi.org/10.1016/j.marpolbul.2004.02.006

SUN, B.; ZHAO, F. J.; LOMBI, E.; MCGRATH, S. P. Leaching of heavy metals from contaminated soils using EDTA. Environmental Pollution, v. 133, n. 2, p. 111-120, 2001. https://doi.org/10.1016/S0269-7491(00)00176-7

UNITED STATES. Environmental Protection Agency - USEPA. Acid mine drainage prediction. Washington DC, 1994.

VALETON, I.; SCHUMANN, A.; VINX, R.; WIENEKE, M. Supergene Alteration Since the Upper Cretaceous on Alkaline Igneous and Metasomatic Rocks of the Poços de Caldas Ring Complex, Minas Gerais, Brazil. Applied Geochemistry, v. 12, p.133-154, 1997. https://doi.org/10.1016/S0883-2927(96)00060-1

VALLE, L. A. R. do. Avaliação de Elementos-Traço em Fertilizantes e Corretivos. 2012. 75f. Dissertação (Mestrado em Ciência do Solo) - Universidade Federal de Lavras, Lavras, 2012.

WABER, N.; SCHORSCHER, H. D.; PETERS, T. Hydrothermal and Supergene Uranium Mineralization at the Osamu Utsumi Mine, Poços de Caldas, Minas Gerais, Brazil. Journal of Geochemical Exploration, v. 45, n. 1-3, p. 53-112, 1992. https://doi.org/10.1016/0375-6742(92)90122-O

YOUSAF, M.; LI, J.; LU, J.; REN, T.; CONG, R.; FAHAD, S.; LI, X. Effects of fertilization on crop production and nutrient-supplying capacity under rice-oilseed rape rotation system. Scientific Reports, v. 7, n. 1270, 2017. https://doi.org/10.1038/s41598-01701412-0 